\title{
Dieta de duas espécies de peixes da família Cichlidae (Astronotus ocellatus e Cichla pinima) introduzidos no rio Paraguaçu, Bahia
}

\author{
Luis Rogério Godinho dos Reis * \\ Alexandre Clistenes de Alcântara Santos \\ Universidade Estadual de Feira de Santana, Departamento de Ciências Biológicas \\ Laboratório de Ictiologia (LABICTIO) \\ Avenida Transnordestina, s/nº, CEP 44036-900, Feira de Santana - BA, Brasil \\ * Autor para correspondência \\ luisrogerio2@hotmail.com \\ Submetido em 05/05/2014
}

Submetido em 17/03/2014

Aceito para publicação em 26/09/2014

\section{Resumo}

A análise da dieta de espécies introduzidas permite entender a posição trófica destas na comunidade de peixes, seu grau de ação predatória, e até em que níveis agem como competidores e/ou predadores de outras espécies. Este trabalho visa descrever a dieta do tucunaré, Cichla pinima, e do apanhari, Astronotus ocellatus, espécies oriundas da bacia amazônica e introduzidas no rio Paraguaçu. Noventa e dois espécimes foram analisados e ambas as espécies apresentaram uma dieta diversificada composta por 20 tipos diferentes de itens. Para $C$. pinima, peixes ( $\mathrm{IAi}=35,2 \%)$ insetos Ephemeroptera ( $\mathrm{IAi}=23,69 \%)$ e Moluscos $(\mathrm{IAi}=21,2 \%)$ foram os mais importantes. Astronotus ocellatus, por sua vez, apresentaram insetos Ephemeroptera como altamente predominantes na dieta correspondendo a mais de $98 \%$ do IAi. A alta predominância de insetos na dieta pode estar relacionada ao alto número de juvenis entre os exemplares analisados. A presença de exemplares de peixes nativos nos conteúdos estomacais dos tucunarés merece atenção, pois, a voracidade deste tipo de peixe pode levar a extinção de espécies nativas.

Palavras-chave: Extinção de espécies; Invasão biológica; Predação

\section{Abstract}

Diet of two species of fish family Cichlidae (Astronotus ocellatus and Cichla pinima) introduced in the Paraguaçu River, Bahia. Analysis of the diet of introduced fish species allows us to understand their trophic position in the community, their level of predation, and possibly to identify the if and when they act as competitors and/or predators. This paper aims to describe the diet of the tucunaré, Cichla pinima, and of the apanhari, Astronotus ocellatus. Both species originated from the Amazon basin and were introduced into the Paraguaçu River. Ninety-two specimens were analyzed, and both species showed a diversified diet composed of 20 different food types. For $C$. pinima, fish $(\mathrm{IAi}=35.2 \%)$ Ephemeroptera insects ( $\mathrm{IAi}=31.7 \%)$ and molluscs $(\mathrm{IAi}=21.2 \%)$ were the most important food types. Ephemeropteran insects were highly prevalent in the diet of A. ocellatus, corresponding to over $98 \%$ of IAi. The high prevalence of insects in the diet may be related to the high number of juveniles among the specimens analyzed. The presence of native fish in the stomach contents of tucunarés deserves particular attention, because the voracity of this fish can lead to extinction of native species.

Key words: Biological invasion; Extinction of species; Predation 


\section{Introdução}

As espécies de peixes que ocorrem no Semiárido representam o resultado de processos evolutivos condicionados por fatores climáticos e pelo regime hidrológico da região (ROSA et al., 2003). Entretanto, processos antrópicos, como as alterações ambientais e os programas de erradicação e introdução de espécies, que possivelmente levaram à exclusão de elementos autóctones, levaram a uma modificação na estrutura da fauna original. Atualmente, são registradas cerca de 240 espécies para o bioma, mas acredita-se que este número possa ser ampliado na medida em que aumentarem os esforços na realização de inventários direcionados para as bacias hidrográficas do semiárido (ROSA, 2004).

Matthews (1998) destaca dentre os principais fatores que estruturam comunidades de peixes, a formação das principais características do habitat no tempo geológico; o estabelecimento ou manutenção da estrutura física ou química do hábitat; e modificações abruptas na estrutura ou em características químicas do hábitat durante períodos ativos de mudanças. Dentre estas, o autor cita as duas últimas como as que ocorrem em uma escala de tempo muito menor e tida como normais e aceitáveis dentro de rios. Entretanto, as alterações antropogênicas de grande magnitude e num curto espaço de tempo teriam a capacidade de afetar significativamente as comunidades de peixes.

Um dos fatores que podem interferir na composição e na diversidade das comunidades naturais é a presença de espécies não nativas. A introdução de espécies é apontada como a segunda maior causa de extinção de espécies de peixes (MILLER et al., 1989; MOYLE; LEIDY, 1992), sendo relacionada à várias razões como, introduções propositais para estoque em reservatórios, aquicultura, controle biológico de vetores de doenças, pesca esportiva, estoque de peixes ornamentais e aumento da pesca de anzol (WELCOMME, 1984; CRIVELLI, 1995; LEVER, 1998). Interações diretas entre espécies nativas e exóticas geram como resultado a competição, predação e parasitismo (VITOUSEK et al., 1987). O sucesso da introdução de espécies pode desencadear extinções e iniciar um efeito cascata em toda a comunidade (PIMM, 1987).
O tucunaré, Cichla pinima é uma espécie da família Cichlidae que pode chegar a $75 \mathrm{~cm}$ de comprimento total. Ocorre no baixo rio Amazonas no Brasil (Tapajós, Curuá-Una, Xingu), e no baixo Tocantins e rios Capim. Exemplares da espécie foram registrados também no Amapá, Araguari, Canumã e de forma introduzida no Rio Paraguaçu, no sudeste do Brasil (KULLANDER et al., 2006). O tucunaré é um peixe piscívoro típico, que causa impacto direto observável nas populações nativas de peixes carnívoros, e, indiretamente, em outros níveis tróficos como, os peixes consumidos pelo predador e, até mesmo pássaros ictiófagos, insetos e os zooplâncton (ZARET; PAINE, 1973).

Astronotus ocellatus, conhecido por apanhari ou Oscar, pertence também à família Cichlidae, podendo chegar ao máximo de $21 \mathrm{~cm}$ de comprimento total. A espécie é nativa da bacia Amazônica e está distribuída em diversos países da América do Sul, como no Brasil, Peru, Colômbia, e Guiana Francesa. Possui valor comercial no mundo inteiro como um peixe ornamental (FRACALOSSI, 2008). A dieta natural do Oscar inclui frutos, moluscos e pequenos peixes, sendo considerado onívoro, com tendência à carnivoria (FERREIRA, 1981). Esta espécie apresenta vários nomes vernaculares locais como Corro Chinês, Corro Baiano, Dorminhoco e Paya. Latini e Petrere (2004) relataram uma significativa diminuição na riqueza de espécies e diversidade da fauna de peixes lacustres no sudeste Brasil após sua introdução.

Robins (2007), por sua vez, expressou a preocupação de que a propagação da espécie introduzida no sul da Flórida pode ter um efeito negativo sobre peixes nativos que têm habitat e necessidades ecológicas semelhantes.

O estudo da dieta e da interação das espécies com o meio fornece importantes informações sobre a ecologia trófica e o comportamento de peixes, diante das variações nas condições ambientais e do alimento disponível. Além disso, fornece subsídios para compreensão de mecanismos que permitem a coexistência e exploração dos recursos de um mesmo sistema por várias espécies (GOULDING, 1981). A análise de sobreposição alimentar das dietas, por exemplo, pode ser utilizada para determinação da ocorrência e intensidade de competição entre as espécies (ZARET; RAND, 1971). 
Nesse sentido, a análise de conteúdo estomacal tem constituído um bom mecanismo no conhecimento dos fatores que conduzem à estruturação de comunidades aquáticas. Assim, o conhecimento das fontes alimentares utilizadas pelos peixes pode fornecer dados sobre habitat, disponibilidade de alimento no ambiente e mesmo sobre alguns aspectos do comportamento (HAHN et al., 1997; GASPAR DA LUZ et al., 2001).

Particularmente em ambientes onde ocorreu introdução de espécies predadoras não nativas, altamente adaptáveis e de rápida proliferação, causa sérios danos às comunidades ícticas nativas, especialmente por interações biológicas como predação e competição e pelos efeitos em cascata na cadeia trófica (GOMIERO, 2004).

Desta forma, o presente trabalho visa analisar a dieta de A.ocellatus e C. pinima, duas espécies de ciclídeos introduzidas no rio Paraguaçu.

\section{Material e Método}

O rio Paraguaçu nasce na Serra do Sincorá, no município de Barra da Estiva, à aproximadamente 1.200 $\mathrm{m}$ de altitude. Percorre cerca de $600 \mathrm{~km}$ até a sua foz na porção oeste da Baía de Todos os Santos, formando antes a Baía de Iguape. Localiza-se na região Centro-Oeste do estado da Bahia, entre as coordenadas de $11^{\circ} 17^{\prime} \mathrm{S}$ e $13^{\circ} 36^{\prime} \mathrm{S}$ de latitude sul, e $38^{\circ} 50^{\prime} \mathrm{W}$ e $42^{\circ} 01^{\prime} \mathrm{W}$ de longitude Oeste (CEPLAB, 1979) (Figura 1).

É o maior rio exclusivamente baiano, apresentando regime fluvial bem variável, valores de pluviosidade entre 500 e $1.200 \mathrm{~mm}$ anuais. Seu curso superior caracterizase por uma pluviometria abundante, com médias anuais superiores a $1.000 \mathrm{~mm}$. A escarpa oriental da Chapada Diamantina, onde se localizam suas nascentes, apresenta um regime torrencial, com enchentes rápidas e violentas que influenciam consideravelmente a dinâmica fluvial. Em sua parte média os índices pluviométricos são mais

FIGURA 1: Mapa do trecho estudado no rio Paraguaçu e os pontos de coleta.

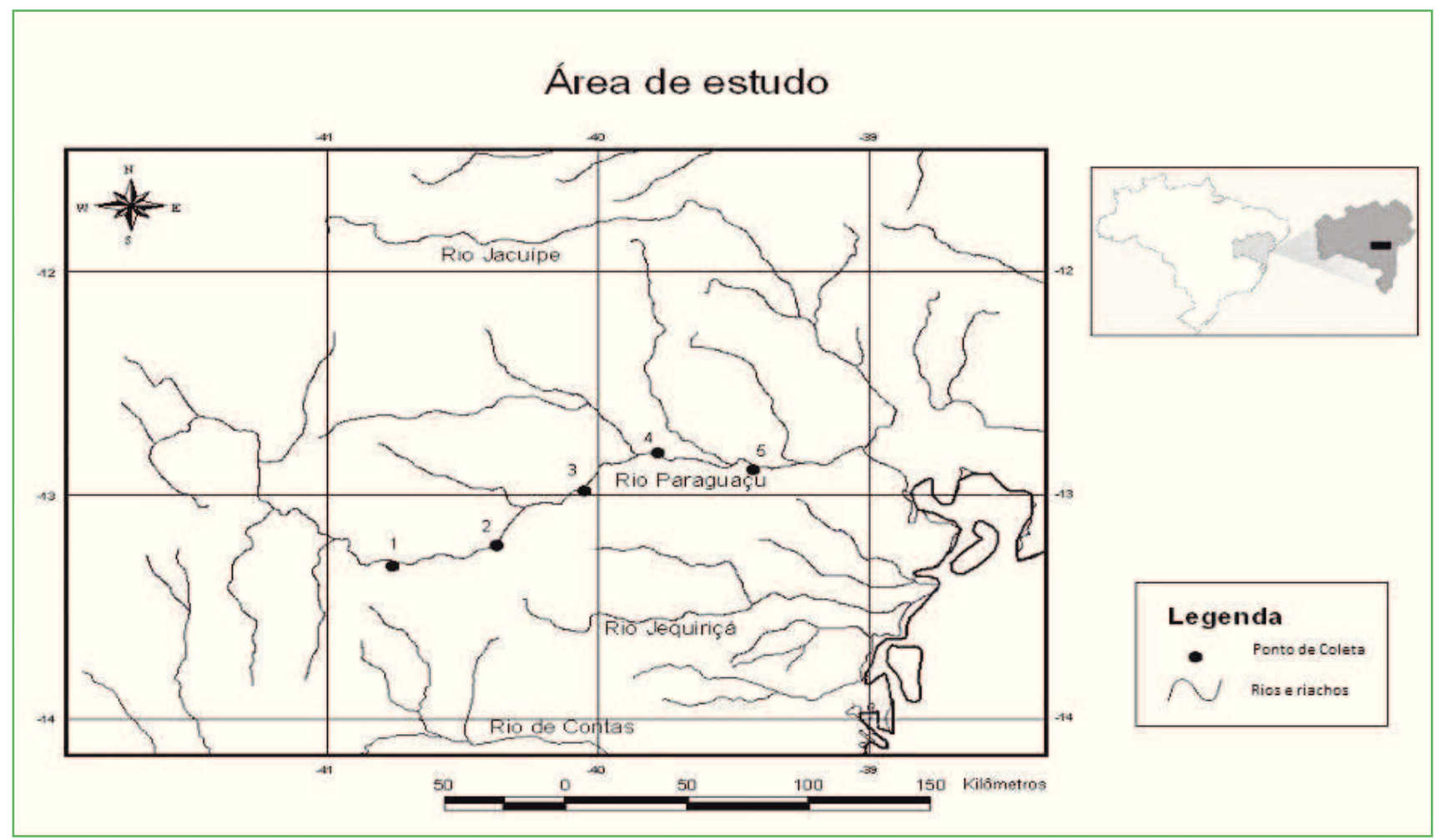


baixos atingindo médias anuais em torno de 500 a $900 \mathrm{~mm}$. A presença de serras a montante deste trecho cria obstáculos à penetração das massas de ar úmidas registradas no centro sul da bacia, particularmente nos meses de agosto e setembro. Com índices mínimos de aproximadamente $10 \mathrm{~mm}$. O curso inferior do rio Paraguaçu apresenta maior regularidade na distribuição de chuvas, com uma variação média mensal de 50 a 100 $\mathrm{mm}$, registrando-se valores mais baixos nos meses de setembro e outubro (CEPLAB, 1979).

Moura (2008) registrou 42 espécies de peixes para o médio curso do rio Paraguaçu, região de realização deste trabalho, sendo que C. pinima e A. ocellatus destacaramse entre as espécies dominantes em redes-de-espera e tarrafa, respectivamente. Estas espécies pertencem à família Cichlidae e foram estudadas no presente trabalho, sendo ambas alóctones, ou não nativas, ou seja, trazidas de outra bacia hidrográfica, de um mesmo país (IBAMA, 1998).

O interesse pela região tem aumentado nos últimos tempos e alguns trabalhos em relação à ecologia de sua ictiofauna têm surgido (SANTOS, 2003; SANTOS; CARAMASCHI, 2007; SANTOS; CARAMASCHI, 2011; MOURA, 2008), porém, há ainda pouca quantidade de dados o que evidencia a carência de informações sobre a bacia e ictiofauna.

Cinco pontos foram amostrados no trecho médio do rio Paraguaçu, tendo sido selecionados de forma aproximadamente equidistante em $50 \mathrm{~km}$, visando uma abrangência representativa da Área de estudo. Os pontos localizaram-se nos distritos de Bandeira de Melo, Marcionílio Souza, Iaçu, Rafael Jambeiro e Castro Alves. Foram realizadas seis expedições de campo entre dezembro de 2005 e maio de 2007, amostrando as estações seca e chuvosa com coletas padronizadas. A definição das estações seca e chuvosa foi baseada em dados acumulados de pluviosidade no período de 1997 a 2007 pelo Instituto Nacional de Meteorologia - INMET. Com o objetivo de amostrar os diferentes microhabitats e maximizar a captura das duas espécies analisadas, foram empregados diferentes métodos de coleta, constituídos de três artes de pesca usadas de forma padronizada: picaré, por período de $1 \mathrm{~h}$, três redes-de-espera de malhas 40, 50 e $60 \mathrm{~mm}$ entre nós, e tarrafa pelo período de $1 \mathrm{~h}$.
Além disso, foram também adquiridos exemplares de pescadores locais.

Os peixes coletados foram imediatamente fixados em solução de formalina a 10\%. Após um mínimo de $48 \mathrm{~h}$, em laboratório, os espécimes foram transferidos para solução de álcool a 70\%.

Os conteúdos estomacais foram retirados e o material foi triado e identificado ao nível taxonômico mais baixo possível, utilizando chaves de identificação específicas para cada grupo (BARNES, 1984; POUGH et al., 1993; COSTA et al., 2006; MUGNAI et al., 2010). Para caracterização da dieta das duas espécies, a análise dos itens do conteúdo estomacal foi feita através do método volumétrico, sob lupa estereoscópica com o auxílio de uma placa quadrada de vidro com bordas de $1 \mathrm{~mm}$ de altura e uma escala milimetrada colocada embaixo, conforme descrito em Albrecht e Caramaschi (2003) para medida do volume.

Para padronizar a listagem de itens visando análise por comparações gráficas e classificação em relação ao grupo trófico, os mesmos foram agrupados em categorias:

Peixe: Peixe, larvas de peixe, escamas e fragmento de peixe.

Molusco: Conchas de Bivalves.

Matéria Inorgânica: Lama, Areia, Sedimento e Pedra.

Insetos Aquáticos: Larva de Odonata, Larva Ephemeroptera, Larva de Trichoptera

Insetos Terrestres: Coleoptera, Diptera, Hymenoptera, Hemiptera, Blataria, Orthoptera.

Outros: Representação gráfica para os itens agrupados que não representaram valores relevantes em relação ao IAI.

A análise da dieta foi realizada através de método qualitativo de Frequência de ocorrência e volumétrico (HYNES, 1950; HYSLOP, 1980; CORTÉS, 1997) combinados no Índice Alimentar (IAi) (KAWAKAMI; VAZZOLER, 1980). Foi também investigada a amplitude do nicho trófico, que pode ser referida como a diversidade de presas consumidas (MAGURRAN, 
1988), avaliada através do índice de diversidade de Shannon (KREBS, 1989). O índice de Shannon é calculado a partir da seguinte equação: $H(n i / N) \log$ $(n i / N)$; onde: $n i=$ valor do IAi de cada item e $\mathrm{N}=$ soma total das frequências de Ocorrência (FO\%). As diferenças significativas na amplitude da dieta entre as espécies foram verificadas através do teste $t$ de Student modificado por (ZAR, 1984), calculado a partir da fórmula: $\mathrm{t}=\mathrm{H}$ '1 - H'2/SH'1 - H'2, onde H' é o índice de diversidade de Shannon e $\mathrm{S}$ é igual a variância dos valores de diversidade entre as amostras.

\section{Resultado e Discussão}

Noventa e dois espécimes de C. pinima (55) e $A$. ocellatus (37) foram analisados e ambas as espécies apresentaram uma dieta composta por 20 itens alimentares distribuídos em oito categorias: decápodas, moluscos, peixe, insetos Ephemeroptera e Hemiptera, outros insetos e outros. As análises identificaram uma variação entre as espécies, revelando uma maior amplitude da dieta em $C$. pinima, em relação à $A$. ocellatus.

Os itens alimentares de maior frequência de ocorrência para $C$. pinima foram insetos das ordens Ephemeroptera e Hemiptera, com 31,7\% e 14,6 $\%$, respectivamente. Para $A$. ocellatus, os valores de frequência de ocorrência foram de $42,7 \%$ para Ephemeroptera e 33,3\% para Hemiptera.
De acordo com o teste de Hutcheson, a amplitude da dieta foi significativamente maior $(\mathrm{p}<0,05)$ em $C$. pinima $\left(\mathrm{H}^{\prime}=2,304\right)$, que em $A$. ocellatus $\left(\mathrm{H}^{\prime}=1,56\right)$ (Tabela 1).

TABELA 1: Resumo estatístico do teste de Hutcheson para a amplitude da dieta entre Cichla pinima e Astronotus ocellatus.

\begin{tabular}{lcc}
\hline \multicolumn{1}{c}{ Espécie } & \multicolumn{2}{c}{$\begin{array}{c}\text { Cichla pinima } \boldsymbol{x} \text { Astronotus } \\
\text { ocellatus }\end{array}$} \\
\hline Cichla pinima & $\mathrm{S}^{2} \mathrm{H}^{\prime} 1$ & 0,0024118 \\
Astronotus ocellattus & $\mathrm{S}^{2} \mathrm{H}^{\prime} 2$ & 0,0032602 \\
& $t$ & 9,878 \\
& $t$ crít. $(\mathrm{p}=0,05)$ & 4,303 \\
\hline
\end{tabular}

O C. pinima apresentou diversos itens alimentares importantes na dieta com maiores valores de IAi (Índice Alimentar) sendo representados por peixes $(\mathrm{IAi}=35,16 \%)$ insetos Ephemeroptera (IAi=23,69\%) e moluscos bivalves (IAi=21,24\%) (Tabela 2). $A$. ocellatus, por sua vez, apresentou insetos Ephemeroptera como altamente predominantes na dieta correspondendo a mais de $98 \%$ do IAi (Tabela 2).

$\mathrm{O}$ alto índice de importância alimentar para a categoria peixes, somado à alta importância também registrada para as categorias Ephemeroptera e moluscos bivalves ratifica o hábito alimentar carnívoro de $C$. pinima (Figura 2).

FIGURA 2: Frequência de ocorrência (FO\%) e do Volume (VO\%) organizados no Índice Alimentar (IAi) das categorias alimentares de Cichla pinima.

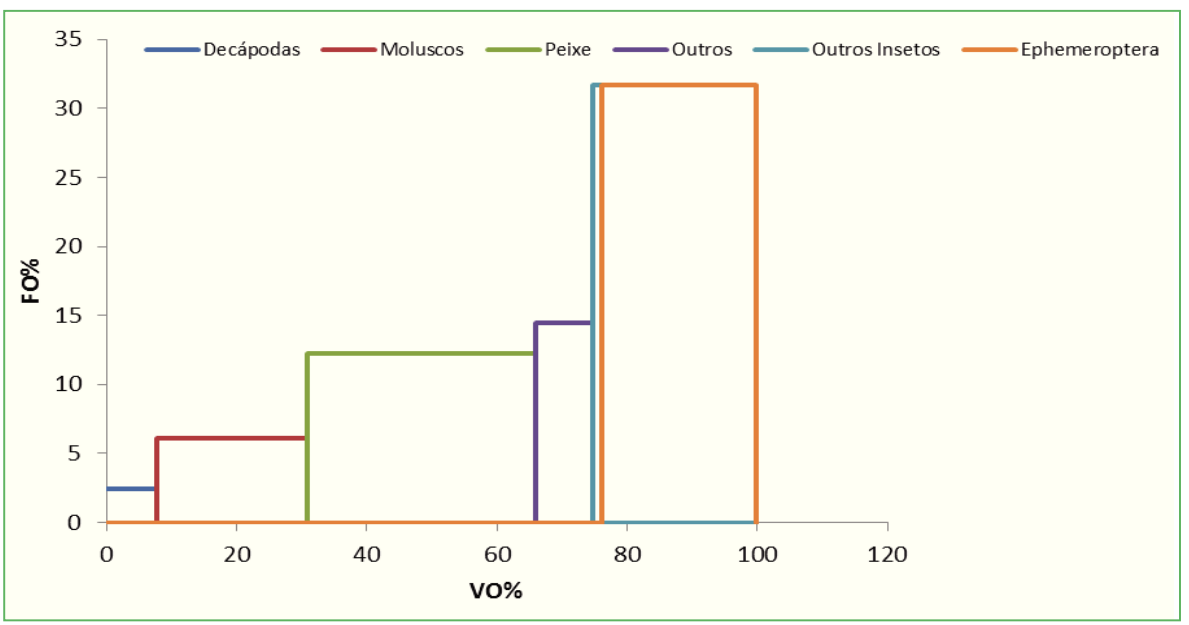


TABELA 2: Frequência de ocorrência e Índice de Importância Alimentar de Cichla pinima e Astronotus ocellatus.

\begin{tabular}{lcccccc}
\hline \multirow{2}{*}{ ITEM } & \multicolumn{3}{c}{ Cichla pinima } & \multicolumn{3}{c}{ Astronotus ocellatus } \\
\cline { 2 - 7 } & FO & FoxVo & IAi & FO & FoxVo & IAi \\
\hline Algae & 0,00 & $<0,01$ & $<0,01$ & 2,7 & 1,03 & 0,03 \\
Araneae & 1,2 & $<0,01$ & $<0,01$ & 1,3 & 4,25 & 0,13 \\
Areia & 1,2 & $<0,01$ & $<0,01$ & 1,3 & 0,04 & 0,00 \\
Bivalvia & 3,7 & 129,32 & 21,24 & 0,00 & 0,00 & 0,00 \\
Blataria & 0,00 & 0,00 & 0,00 & 1,3 & 0,04 & 0,00 \\
Cerdas & 2,4 & 0,02 & $<0,01$ & 0,00 & 0,00 & 0,00 \\
Coleoptera & 3,7 & $<0,01$ & $<0,01$ & 1,3 & 3,89 & 0,12 \\
Decapoda & 2,4 & 47,33 & 7,78 & 0,00 & 0,00 & 0,00 \\
Diptera & 1,2 & 0,22 & 0,04 & 0,00 & 0,00 & 0,00 \\
Larva Ephemeroptera & 31,7 & 144,24 & 23,69 & 42,74 & 3057,32 & 98,5 \\
Escamas Cicloides & 2,4 & 0,02 & $<0,01$ & 1,3 & 0,04 & 0,00 \\
Gastrópode & 2,4 & 11,06 & 1,82 & 0,00 & 0,00 & 0,00 \\
Hymenoptera & 4,9 & 4,36 & 0,72 & 0,00 & 0,00 & 0,00 \\
Hemiptera & 14,6 & 2,63 & 0,43 & 33,3 & 17,98 & 0,58 \\
Material Amorfo & 7,3 & 53,14 & 8,73 & 1,3 & 1,42 & 0,04 \\
Material Vegetal & 2,4 & 0,02 & $<0,01$ & 0,00 & 0,00 & 0,00 \\
Larva de Odonata & 1,2 & 0,80 & 0,13 & 1,3 & 8,85 & 0,28 \\
Orthoptera & 6,1 & 1,53 & 0,25 & 6,7 & 1,81 & 0,05 \\
Peixe & 9,8 & 214,03 & 35,16 & 0,00 & 0,00 & 0,00 \\
Trichoptera & 0,00 & 0,00 & 0,00 & 5,3 & 5,78 & 0,18 \\
\hline
\end{tabular}

FIGURA 3: Frequência de ocorrência (FO\%) e do Volume (VO\%) organizados no Índice Alimentar (IAi) das categorias alimentares de Astronotus ocellatus.

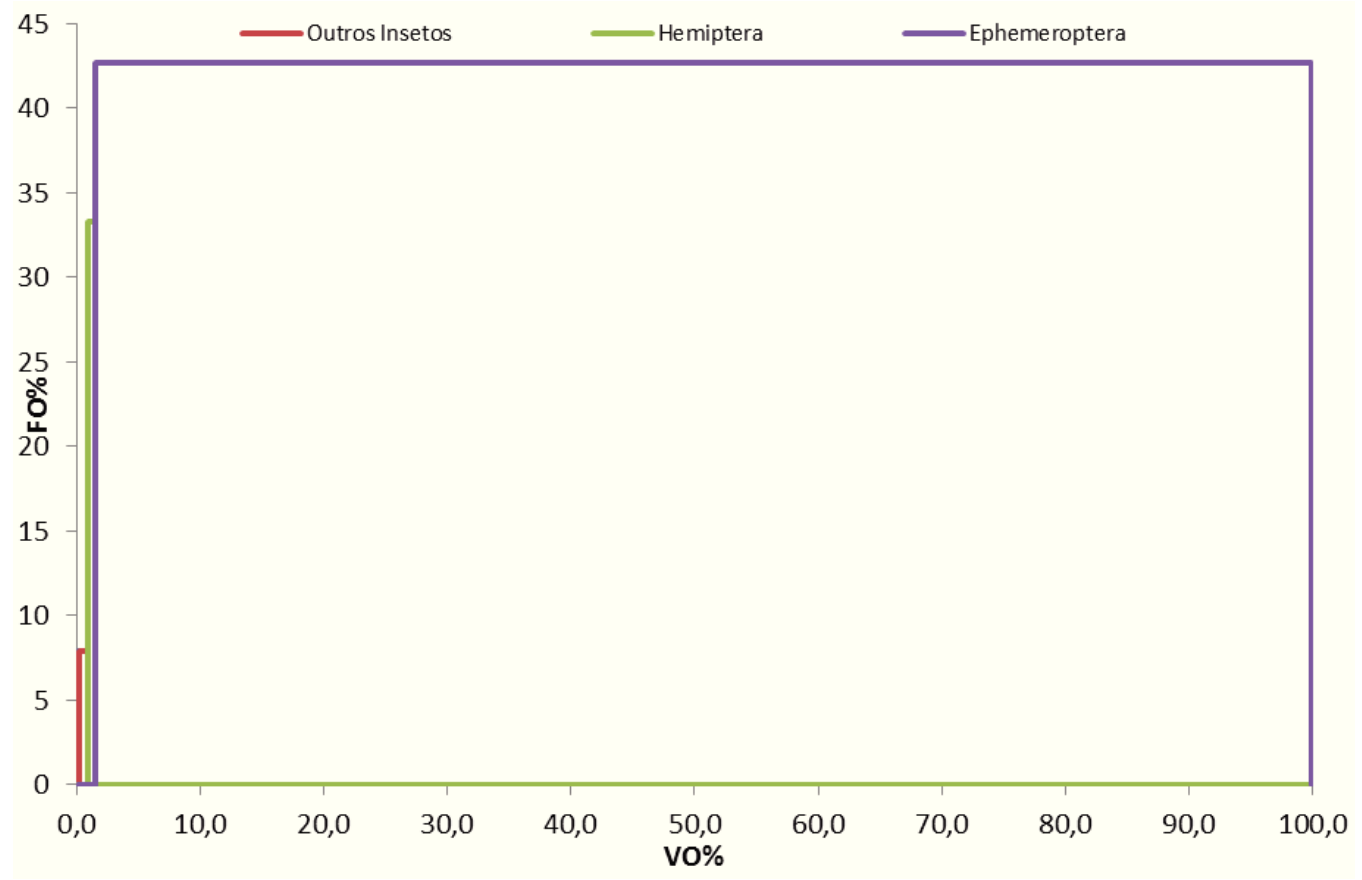


Para A. ocellatus, ao agrupar os itens alimentares em categorias (Figura 3), o item Ephemeroptera mostra-se altamente predominante na dieta, indicando a guilda trófica da espécie como carnívora insetívora. Entretanto, o registro da presença de escamas cicloides nos estômagos destes animais pode indicar a predação de peixes, uma vez que os tecidos moles são digeridos mais rapidamente que estas escamas, e o grau de digestão avançado pode ter dificultado a identificação deste item.

O A. ocellatus tem hábitos noturnos (SANTOS et al., 1984; 2006; SOARES et al., 2008), e quanto ao hábito alimentar, Santos et al. (2006) afirmam que o peixe é onívoro, com tendência à carnivoria, consumindo principalmente peixes, insetos, camarões e eventualmente frutos e sementes. A mesma espécie foi caracterizada também por Ferreira (1981) como onívora com tendências carnívoras, sendo registrada a presença de pequenos peixes na dieta da espécie.

Apesar de serem espécies carnívoras, e da presença de peixes como itens importantes na dieta de C. pinima, valores representativos na categoria Insetos para ambas as espécies podem ser explicados pela predominância de juvenis nas amostras, o que levaria a um aumento da frequência deste item na alimentação. Gomiero e Braga (2004) citam que o gênero Cichla no reservatório de Volta Grande, MG/SP, apresentou notória mudança ontogenética na dieta das duas espécies, sendo que os jovens se alimentaram de crustáceos e insetos e os adultos, principalmente de peixes.

As análises permitiram também identificar a ocorrência de peixes nativos na dieta do tucunaré, como Astyanax sp. e indivíduos da família Anostomidae. Este fato é relevante, pois, indica a predação de espécies nativas podendo levar a uma modificação da estrutura de comunidade do médio curso do rio Paraguaçu causado pela introdução de espécies.

O tucunaré, em particular, é causador de graves alterações nas comunidades de peixes em que foi introduzido, embora sua ação seja, ainda, pouco documentada (ZARET; PAINE, 1973; JEPSEN et al., 1997). Gomiero e Braga (2004), estudando a alimentação do gênero Cichla no reservatório de Volta Grande (MG/ SP) demonstraram que comportamento piscívoro era predominante, apesar de crustáceos e insetos também terem sido registrados na dieta. Luiz et al. (2011), analisando a dieta de C. piquiti no rio Paraíba, demonstraram uma dieta predominantemente piscívora deste peixe em todas as fases do seu crescimento, com fragmentos de plantas, insetos e crustáceos desempenhando um parte menos importante nos hábitos alimentares.

$\mathrm{Na}$ área de estudo, a presença de peixes nativos na dieta das espécies introduzidas já pode ser considerada um fator importante. Trabalhos anteriores apontam registros de mudança da dieta de espécies piscívoras nativas na presença de piscívoros introduzidos (POMPEU; GODINHO, 2001; SANTOS, 2003). Esta modificação estrutural de comunidade, inclusive, já foi relatada e corroborada por pescadores sobre a relação entre a ação predatória do tucunaré na região, e o desaparecimento de espécies nativas como matrinchã e pirá (MOURA, 2002). Este contexto reforça a necessidade da realização de estudos de monitoramento da ictiofauna da região visando detectar evidências de mudanças na estrutura da ictiofauna local causada por introduções.

$\mathrm{Na}$ área em estudo, as duas espécies de ciclídeos apresentaram dieta diversificada com grande número de itens, sendo que $C$. pinima apresentou dieta mais diversificada, com maior número de itens registrado.

Cichla pinima foi considerada carnívora com alto consumo de peixes, moluscos bivalves e insetos, enquanto A. ocellatus foi classificada como carnívora, com alta predominância de insetos na dieta, podendo este fato estar relacionado ao alto número de juvenis entre os exemplares analisados.

Desta forma, é descrita a dieta de dois ciclídeos que vêm sendo usualmente introduzidos em diferentes bacias do continente sul-americano. A presença de exemplares de peixes nativos nos conteúdos estomacais dos tucunarés sugere que possa haver modificações na estrutura da comunidade, que podem inclusive levar a extinção de espécies nativas, conforme já vem sendo relatado na literatura. Neste contexto, os resultados constituem uma contribuição importante para estudos posteriores que avaliem o efeito destas introduções na comunidade de peixes local. 


\section{Referências}

ALBRECHT, M. P.; CARAMASCHI, P. E. Feeding ecology of Leporinus taeniofasciatus (Characiformes: Anostomidae) before and after installation of a hydroelectric plant in the upper rio Tocantins, Brazil. Neotropical Ichthyology, Porto Alegre, v. 1, n. 1, p. 53-60, 2003.

BARNES, R. D. Zoologia dos Invertebrados. 4 ed. Pennsylvania: Rocca, 1984. 1179 p.

CEPLAB. Bacias hidrográficas do estado da Bahia. Séries Recursos Naturais. Salvador: CEPLAB, 1979. 89 p.

COSTA, C.; IDE, S.; SIMONKA, C. E. Insetos imaturos. Metamorfose e Identificação. Ribeirão Preto: Holos, 2006. 249 p.

CORTÉS, E. A critical review of methods of studying fish feeding based on analysis of stomach contents: application to elasmobranch fishes. Canadian Journal of Fisheries and Aquatic Sciences, Toronto, v. 54, p. 726-738, 1997.

CRIVELLI, A. J. Are fish introductions a threat to endemic freshwater fishes in the Northern Mediterranean Region? Biological Conservation, Boston, v. 72, n. 2, p. 311-319, 1995.

FERREIRA, E. J. G. Alimentação dos adultos de doze espécies de cichlídeos (Perciformes, Cichlidae) do rio Negro, Brasil. 1981. 254 f. Dissertação (Mestrado em Biologia de Água Doce e Pesca Interior) - Instituto Nacional de Pesquisas da Amazônia/Fundação Universidade do Amazonas, Manaus. 1981.

FRACALOSSI, D. M.; ALLEN, M. E.; NICHOLS, D. K.; OFTEDAL, O. T. Oscars, Astronotus ocellatus, have a dietary requirement for vitamin C. The Journal of Nutrition,

Cambridge, v. 128, n. 10, p. 1745-1751, 1998.

GASPAR DA LUZ, K. D.; ABUJANRA, F.; AGOSTINHO, A. A.; GOMES, L. C. Caracterização trófica da ictiofauna de três lagoas da planície aluvial do alto rio Paraná, Brasil. Acta Scientiarum, Maringá, v. 23, n. 2, p. 401- 407, 2001.

GOMIERO, L. M.; BRAGA, F. M. S. Feeding of introduced species of Cichla (Perciformes, Cichlidae) in Volta Grande Reservoir, river grande (Mg/Sp). Brazilian Journal of Biology, São Carlos, v. 64, n. 4, p. 787-795, 2004.

GOULDING, M. Man and fisheries on an Amazon frontier. Vol. 4. Dumont: Developments in Hydrobiology, 1981. 137 p.

HAHN, N. S.; ADRIAN, I. F.; FUGI, R.; ALMEIDA, V. L. L. Ecologia trófica. In: VAZZOLER, A. E. A. M.; AGOSTINHO, A. A.; HAHN, N. S. (Ed.). A planície de inundação do Alto Rio Paraná: aspectos físicos, biológicos e socioeconômicos. Maringá: EDUEM, 1997. p. 209-228.

HYNES, H. B. N. The food of freshwater sticklebacks (Gasterosteus aculeatus and Pygosteus pungitius) with a review of methods used in studies of the food of fishes. Journal of Animal Ecology, Oxford, v. 19, p. 36-58, 1950.

HYSLOP, E. J. Stomach contents analysis - a review of methods and their application. Journal of Fish Biology, Malden, v. 17, p. 411-42, 1980.

IBAMA. Portaria IBAMA № 145-N. 1998. Disponível em < http:// www.ibama.gov.br/sophia/cnia/legislacao/IBAMA/PT0145181001.pdf $>$.

JEPSEN, D. B.; WINEMILLER, K. O.; TAPHORN, D. C. Temporal patterns of resource partitioning among Cichla species in a Venezuela Blackwater River. Journal of Fish Biology, Malden, v. 51, p. 1085-1108, 1997.

KAWAKAMI, E.; VAZZOLER, G. Método gráfico e estimativa de índice alimentar aplicado no estudo de alimentação de peixes. Boletim Instituto Oceanográfico, São Paulo, v. 29, p. 205-207, 1980.

KREBS, C. J. Ecological metodology. New York: Harper Collins Publishers, 1989. 654 p.

KULLANDER, S. O.; FERREIRA, E. J. G.; EFREM, J. G. A review of the South American cichlid genus Cichla, with descriptions of nine new species (Teleostei: Cichlidae). Ichthyological Exploration of Freshwaters, München, v. 17, n. 4, p. 289-398, 2006.

LATINI, A. O.; PETRERE, M. Reduction of a native fish fauna by alien species: an example from Brazilian freshwater tropical lakes. Fisheries Management and Ecology, East Yorkshire, v. 11, p. 7179,2004

LEVER, C. Introduced fishes: an overview. In: Cowx, I. G. (Ed.). Stocking and introduction of fish. Oxford: Fishing News Books, 1998. p. 143-152.

LUIZ, F. T.; VELLUDO, M. R.; PERET, A. C.; RODRIGUES FILHO, J. L.; PERET, A. M. Diet, reproduction and population structure of the introduced Amazonian fish Cichla piquiti (Perciformes: Cichlidae) in the Cachoeira Dourada reservoir (Paranaíba River,central Brazil). Revista de Biología Tropical, San José, v. 59, n. 2, p. 727-741, 2011.

MAGURRAN, A. E. Ecological diversity and its measurements. Princeton: Princeton University, 1988. 179 p.

MATTHEWS, W. J. Patterns in freshwater fish ecology. New York: Chapman \& Hall, 1998. 756 p.

MILlER, R. R.; WILliamS, J. D.; WILliAMS, J. E. Extinctions of North American fishes During the past century. Fisheries, Bethesda, v. 14, n. 6, p. 22-37, 1989.

MOURA, F. B. P. Entre o peixe e o dendê: etnoecologia do povo dos Marimbus (Chapada Diamantina, BA). 2002. 121 f. Tese (Doutorado em Ecologia e Recursos Naturais) - Universidade Federal de São Carlos, São Carlos. 2002.

MOURA, P. E. S. Aspectos ecológicos da ictiofauna do médio curso do rio Paraguaçu, Bahia, Brasil. 2008. 31 f. Dissertação (Mestrado em Ecologia) - Universidade Estadual de Feira de Santana, Feira de Santana. 2008

MOYLE, P. B.; LEIDY, R. A. Loss of biodiversity in aquatic ecosystems: evidence from fish faunas. In: FIELDER P. L.; JAIN S. K. (Ed.). Conservation biology: the theory and practice of nature conservation, preservation and management. New York: Chapman and Hall, 1992. p. 127-169.

MUGNAI, R.; NESSIMIAN, J. L.; BAPTISTA, D. F. Manual de identificação de macroinvertebrados aquáticos do estado do Rio de Janeiro. Rio de Janeiro: Technical Books, 2010. p. 173.

PIMM, S. L. Determining the effects of introduced species. Tree, Vancouver, v. 2, n. 4, p. 106-108, 1987

POUGH, F. H.; HEISER, J. B.; MCFARLAND, W. N. A vida dos vertebrados. São Paulo: Atheneu, 1993. 876 p.

POMPEU, P. S.; GODINHO, A. L. Mudança na dieta da traíra Hoplias malaaricus (Bloch) (Erythrinidae, Characiformes) em lagoas da bacia do rio doce devido à introdução de peixes 
piscívoros. Revista Brasileira de Zoologia, Curitiba, v. 18, n. 4, p. 1219-1225, 2001.

ROBINS, R. H. Oscar. Education webpage for Ichthyology at the Florida Museum of Natural History. 2007. Disponível em: <http:// www.flmnh.ufl.edu/fish/>.

ROSA, R. S. Diversidade e conservação dos peixes da caatinga. In: DA SILVA, J. M.; TABARELLI, M.; FONSECA, M. T.; LINS, L. V. (Ed.). Biodiversidade da Caatinga: áreas e ações prioritárias para a conservação. Brasília: Ministério do Meio Ambiente, 2004. p. 382.

ROSA, R. S.; MENEZES, N. A.; BRITSKI, H. A.; COSTA, W. J. E. M.; GROTH, F. Diversidade, padrões de distribuição e conservação dos peixes da Caatinga. In: LEAL, I. R.; TABARELLI, M.; SILVA, J. M. C. Ecologia e conservação da Caatinga. Recife: Editora Universitária, 2003. p. 135-180.

SANTOS, A. C. A. Caracterização da ictiofauna do alto rio Paraguaçu, com ênfase nos rios Santo Antônio e São José, Chapada Diamantina, Bahia. 2003. 215 f. Tese (Doutorado em Ecologia) - Universidade Federal do Rio de Janeiro/Museu Nacional, Rio de Janeiro. 2003.

SANTOS, A. C. de A.; CARAMASCHI, E. P. Composition and Seasonal Variation of the Ichthyofauna from Upper Rio Paraguaçu (Chapada Diamantina, Bahia, Brazil). Archives of Biology and Technology, Curitiba, v. 50, n. 4, p. 663-672, 2007.

SANTOS, A. C. de A.; CARAMASCHI, E. P. Temporal variation in fish composition and abundance in a perennial tributary of the rio Paraguaçu, a little-known drainage in the Brazilian semiarid region. Neotropical Ichthyology, Porto Alegre, v. 9, n. 1, s/ paginação, 2011.
SANTOS, G. M.; JEGU, M.; MERONA, B. Catálogo de peixes comerciais do baixo rio Tocantins. Manaus: ELETRONORTE/ CNPq/INPA, 1984. 86 p.

SANTOS, G. M.; FERREIRA, E. J. G.; ZUANON, J. A. S. Peixes comerciais de Manaus. Manaus: ProVárzea/IBAMA/AM, 2006. $18 \mathrm{p}$.

SOARES, M. G. M.; COSTA, E. L.; SIQUEIRA-SOUZA, F. K.; ANJOS, H. D. B.; YAMAMOTO, K. C.; CARVALHO FREITAS, C. E. C. Peixes de lagos do Médio Rio Solimões. 2 ed. Manaus: Instituto Piatam-I, 2008. 160 p.

VITOUSEK, P. M.; WALKER, L. R.; WHITEAKER, L. D.; MUELLER-DOMBOIS, D.; MATSON, P. A. Biological invasion by Myrica faya alters ecosystem development in Hawaii. Science, New York, v. 238, n. 4828, p. 802-804, 1987.

WELCOMME, R. L. International transfers of Inland fish species. In: COURTENAY, W. R.; STAUFFER, J. R. (Ed.). Distribution, biology, and management of exotic fishes. California: University Press, 1984. p. 22-40.

ZAR, J. H. Biostatistical analysis. New Jersey: Prentice Hall, 1984. $663 \mathrm{p}$.

ZARET, T. M.; PAINE, T. R. T. Species introduction in a tropical lake. Science, New York, v. 182, p. 445-449, 1973.

ZARET, N. T.; RAND, A. S. Competition in tropical stream fishes: support for the competitive exclusion principle. Ecology, New York, v. 52, n. 2, p. 336-342, 1971. 